\title{
Oligothiophene-Bridged Conjugated Covalent Organic Frameworks
}

\author{
Niklas Keller, ${ }^{\dagger}$ Derya Bessinger, ${ }^{\dagger}$ Stephan Reuter, ${ }^{\dagger}$ Mona Calik, ${ }^{\dagger}$ Laura Ascherl, ${ }^{\dagger}$ Fabian C. Hanusch, ${ }^{\dagger}$ \\ Florian Auras, $*, \dagger,+$ and Thomas Bein $*, \dagger$ \\ ${ }^{\dagger}$ Department of Chemistry and Center for NanoScience (CeNS), University of Munich (LMU), Butenandtstraße 5-13, 81377 \\ Munich, Germany \\ ${ }^{\ddagger}$ Cavendish Laboratory, University of Cambridge, Cambridge CB3 OHE, United Kingdom
}

\section{Supporting Information}

ABSTRACT: Two-dimensional covalent organic frameworks (2D-COFs) are crystalline, porous materials comprising aligned columns of $\pi$-stacked building blocks. With a view toward the application of these materials in organic electronics and optoelectronics, the construction of oligothiophene-based COFs would be highly desirable. The realization of such materials, however, has remained a challenge, in particular with respect to laterally conjugated imine-linked COFs. We have developed a new building block design employing an asymmetric modification on an otherwise symmetric backbone that allows us to construct a series of highly crystalline quaterthiophene-derived COFs with tunable electronic proper-

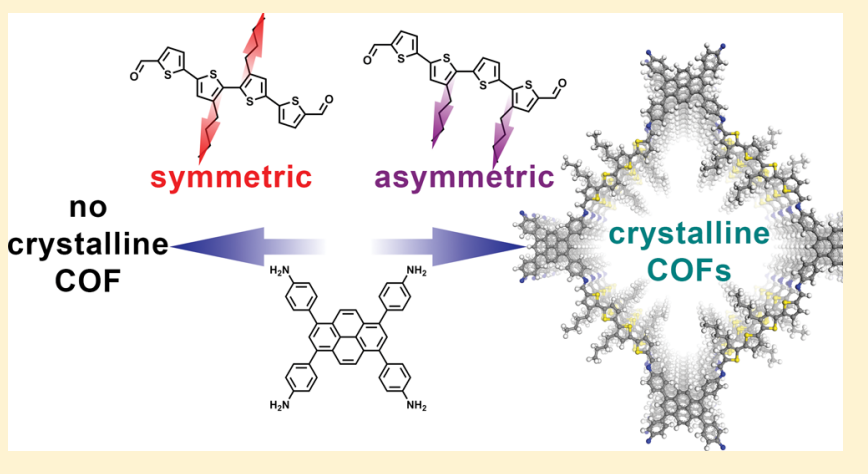
ties. Studying the optical response of these materials, we have observed for the first time the formation of a charge transfer state between the COF subunits across the imine bond. We believe that our new building block design provides a general strategy for the construction of well-ordered COFs from various extended building blocks, thus greatly expanding the range of applicable molecules.

\section{INTRODUCTION}

Since the development of the first covalent organic frameworks (COFs) in 2005, ${ }^{1}$ these materials have attracted considerable scientific interest for applications in gas storage and separation, ${ }^{2,3}$ in photocatalysis, ${ }^{4,5}$ as proton conducting materials, ${ }^{6,7}$ and in optoelectronics. ${ }^{8,9}$ Cross-linking multidentate organic building blocks via reversible covalent bond formation into a crystalline porous framework allows for the construction of robust materials with tunable functionality based on the selection of their building blocks. ${ }^{10-16}$ This way, a great variety of highly ordered materials can be realized having specific combinations of properties that can offer advantages over already established materials, such as zeolites or conductive polymers. With a view on optoelectronic properties, sheet-like $2 \mathrm{D}$-COFs are of particular interest. ${ }^{17}$ In these materials, the covalently linked layers are tightly $\pi$-stacked, thus forming columns with significant electronic overlap that enable efficient migration of excitations and charge carriers. $^{18-20}$

Oligo- and polythiophene-based materials, such as $2,2^{\prime}: 5^{\prime}, 2^{\prime \prime}: 5^{\prime \prime}, 2^{\prime \prime \prime}$-quaterthiophene (4T), $\alpha$-sexithiophene (6T), and poly(3-hexylthiophene-2,5-diyl) (P3HT), are among the most prominent molecular semiconductors in organic field effect transistors (OFETs) and organic photovoltaics (OPV). ${ }^{21-23}$ These materials benefit from high intrinsic charge carrier mobilities of both electrons and holes, typically broad optical absorption throughout the visible spectrum, and good chemical stability. ${ }^{24-26}$

The incorporation of this important class of molecular semiconductors into a COF, however, has remained a challenge. Only the smallest thiophene-based building blocks, i.e., thiophene, bithiophene $(2 \mathrm{~T}),{ }^{27}$ and small fused thiophenes such as thieno[3,2- $b]$ thiophene $(\mathrm{TT})^{8,11}$ and benzo[1,2- $b: 4,5-$ $b^{\prime}$ dithiophene (BDT), ${ }^{28-30}$ have been applied as COF building blocks to date, and mostly in boronate-linked COFs that lack electronic conjugation within the COF layers.

With a view on COFs for optoelectronics, it would thus be highly desirable to develop a method to integrate larger oligothiophenes $(n \mathrm{~T}, n \geq 4)$ that allow for significant $\pi$-overlap and spectral coverage into a conjugated COF.

As flat, extended conjugated building blocks typically suffer from very low solubility in all common solvents, solubilizing groups such as alkyl chains are required for tuning the solubility into the optimal regime for COF growth. ${ }^{30,31}$ However, even short alkyl chains can severely impede the close face-on oriented packing that is a prerequisite for the formation of conductive 2D-COFs.

In this work, we have developed a building block design concept that allows us to overcome these limitations through an asymmetric functionalization of an otherwise $C_{2}$-symmetric

Received: February 15, 2017

Published: June 6, 2017 


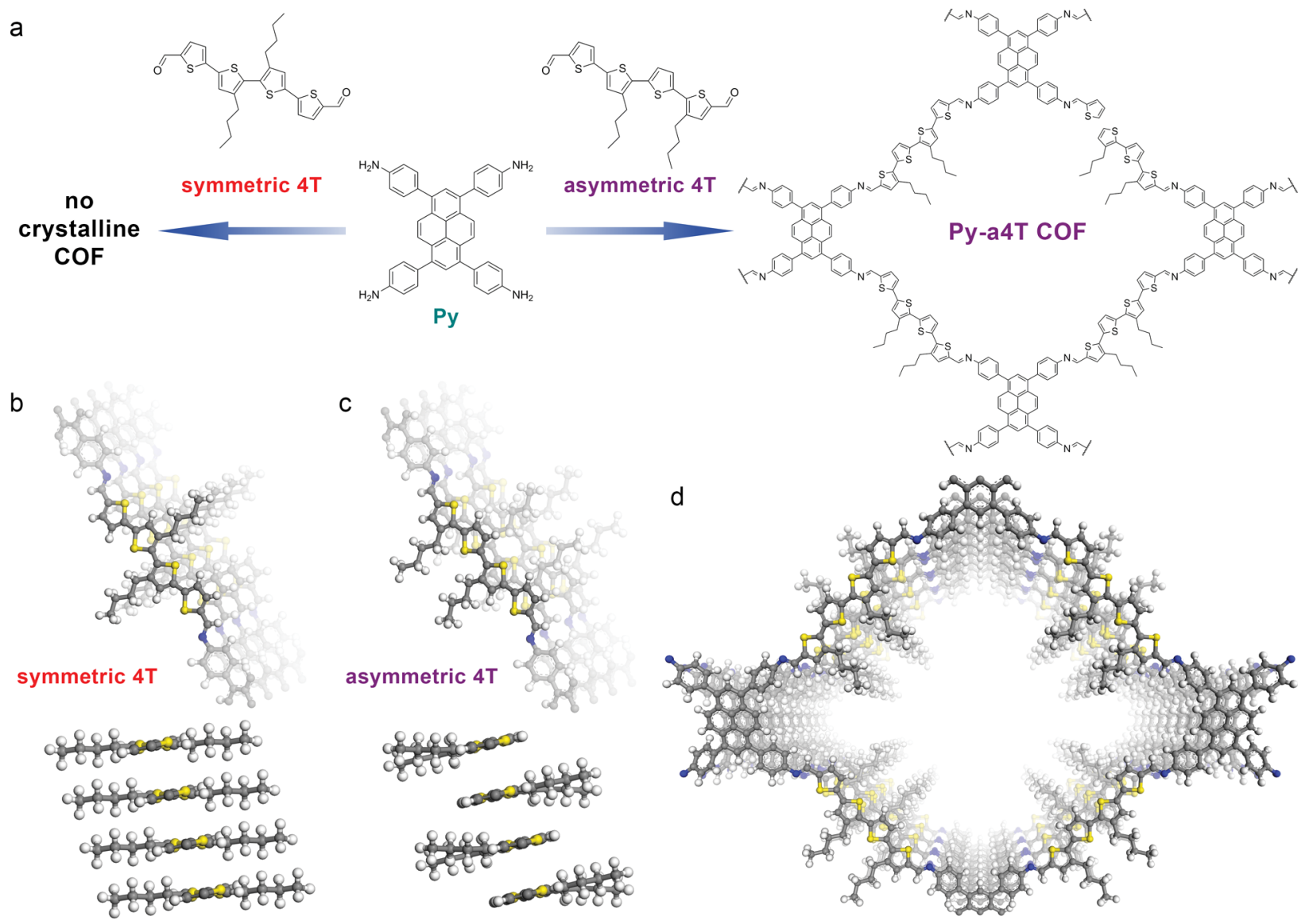

Figure 1. (a) Co-condensation of 1,3,6,8-tetrakis(4-aminophenyl)pyrene (Py) with 2 equiv of the quaterthiophene dialdehyde bearing asymmetric butyl chains (a4T) leads to the formation of the Py-a4T COF. When employing a quaterthiophene dialdehyde with a symmetric configuration of the alkyl chains (s4T), however, no crystalline material can be obtained. (b) Cut-out of the DFT-optimized hypothetical Py-s4T COF structure. Slipped cofacial stacking of the $\mathrm{s} 4 \mathrm{~T}$ building block would place the alkyl chains of adjacent layers in close vicinity, causing considerable steric repulsion and impeding the close packing required for $2 \mathrm{D}-\mathrm{COF}$ formation. (c) This steric repulsion can be avoided by attaching the alkyl chains to the first and third thiophene unit (a4T) instead, allowing for sufficiently spaced alternating alkyl chains while a close-packed cofacial arrangement of the thiophene backbone is supported. (d) DFT-optimized structure of the Py-a4T COF with alternating orientation of the alkyl chains viewed along the pseudoquadratic pore.

4T backbone. This way, we can incorporate solubilizing alkyl chains and allow them to integrate into the COF lattice at sufficiently large distances, while maintaining the desirable close contacts between the thiophene layers. We then extended this concept to electronic modifications of the backbone via the incorporation of thiophene-based acceptor units, such as $4 \mathrm{H}$ thieno[3,4-c]pyrrole-4,6(5H)-dione (TPD) and thieno[3,4b]thiophene (TT). Combining these modified $4 \mathrm{~T}$ building blocks with pyrene-based moieties, we synthesized a first series of highly crystalline quaterthiophene-linked COFs with tunable optical absorption and emission properties.

\section{RESULTS AND DISCUSSION}

Starting from commercially available 3-bromothiophene, we synthesized the quaterthiophene (4T) dialdehyde building blocks via Ni- and Pd-catalyzed Kumada and Suzuki crosscoupling reactions [see the Supporting Information (SI) for details]. In order to study the effect of the configuration of the alkyl chains, we synthesized a symmetric and an asymmetric version of the dibutyl-4T, in which the alkyl chains are attached to the 3-positions of the second and third (s4T) and the first and third thiophene units (a4T), respectively (Figure 1a).
For constructing the imine-linked COFs, we followed a synchronized offset stacking approach. ${ }^{15}$ This COF design uses geometrically interlocked layers for superior crystallinity while enabling close-packed arrangements of both subunits and is perfectly compatible with the quasi $C_{2}$-symmetric flat geometry of the $4 \mathrm{~T}$ bridges. The quaterdentate 1,3,6,8-tetrakis(4aminophenyl)pyrene (Py) has previously been applied in the synthesis of several imine-linked frameworks and has proven to generate stable and exceptionally crystalline 2D-COFs. ${ }^{15,32,33}$ Pyrene-based frameworks feature a quasi-quadratic geometry comprising close-packed, slightly slip-stacked pyrene columns and equally close-packed, slip-stacked linear bridges, thus providing the ideal geometric environment for our new $4 \mathrm{~T}$ building blocks.

On the basis of published pyrene COF structures and density functional theory (DFT) simulations, we expect the 4T unit to be incorporated as face-on oriented stacks, whereby each layer is slipped by about $0.1 \mathrm{~nm}$, mainly perpendicular to the $4 \mathrm{~T}$ axis (Figure $1 \mathrm{~b}, \mathrm{c}$ ). In the case of $\mathrm{s} 4 \mathrm{~T}$, this would bring the alkyl chains of adjacent layers very close together and most likely cause considerable steric repulsion, forcing the framework into a less closely stacked conformation and sacrificing in part the $\pi$ overlap between the thiophenes. On the other hand, a4T can stack with the butyl chains alternating on both sides of the 


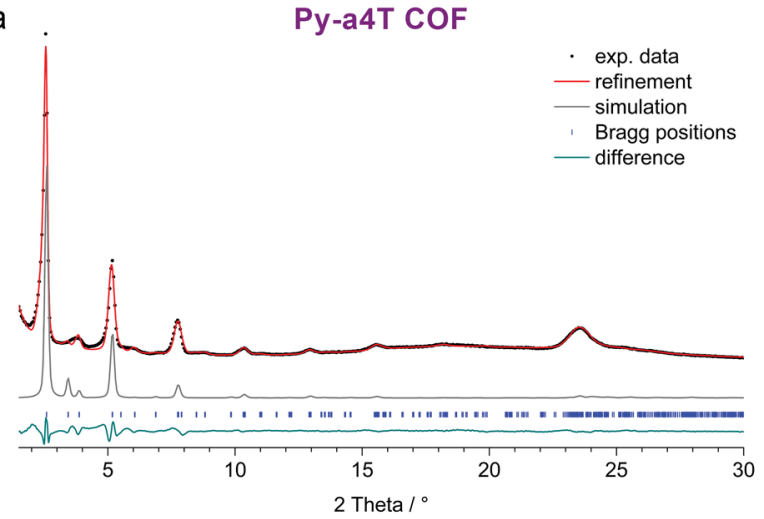

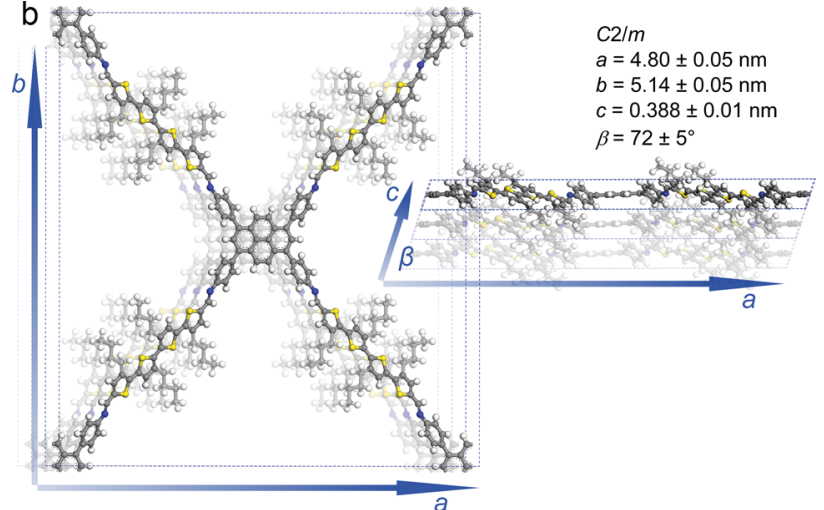

Figure 2. (a) Experimental PXRD data (black dots) of Py-a4T COF. Pawley refinement (red line) using the C2/m symmetric structure model shown in part $\mathrm{b}$ provides a very good fit to the experimental pattern with only minimal differences (the green line shows the difference plot between the experimental and refined patterns; $R_{\mathrm{wp}}=7.5 \%, R_{\mathrm{p}}=13.4 \%$ ). Bragg positions are indicated with blue ticks. The alkyl chains of the a4T building block were treated in the refinement as a superposition of both possible orientations with $50 \%$ occupancy each (both shown in the figure). The simulated PXRD pattern based on the $C 2 / m$ symmetric unit cell (gray line) is in very good agreement with the experimental and the refined pattern. Differences in the peak intensities, in particular the altered intensity ratios between lower- and higher-angle reflections, might stem from small oligomeric fragments that are trapped in the large COF pores (the attenuation of peak intensities due to this will be strongest at small $2 \theta$ angles).

stack, thus allowing for much larger spacing between these alkyl chains while maintaining a close-packed face-on orientation of the $4 \mathrm{~T}$ backbone (Figure 1c,d).

Acid-catalyzed solvothermal synthesis using a4T and Py in a 2:1 molar ratio yielded the anticipated Py-a4T COF as a dark red powder (see the SI for details). The successful formation of a crystalline material was confirmed by powder X-ray diffraction (PXRD; Figure 2a). The presence of multiple higher-order reflections reveals the high degree of long-range order present in this new COF. Pawley refinement using a force-fieldoptimized $\mathrm{C} 2 / \mathrm{m}$ symmetric structure model (Figure 2b) provides a very good fit to the experimental data and produced the lattice parameters $a=4.80 \pm 0.05 \mathrm{~nm}, b=5.14 \pm 0.05 \mathrm{~nm}$, $c=0.388 \pm 0.01 \mathrm{~nm}$, and $\beta=72^{\circ} \pm 5^{\circ}$. From the above discussion and the computational modeling we anticipate an alternating sequence of the alkyl chains as the predominant stacking motif within a single a4T stack. The four a4T stacks that constitute the walls of a COF pore, however, are separated well enough such that they will not form an ordered superstructure. We thus treated the alkyl chains in the Pawley refinement and the simulation of the PXRD pattern as a superposition of both possible orientations with $50 \%$ occupancy each.

The formation of an ordered porous framework was further confirmed by transmission electron microscopy (TEM) (SI, Figure S14). The contrast of the pore channels is visible for a number of crystallites with a projected periodicity of $3.4 \mathrm{~nm}$, which corresponds very well to the channel repeat distance of $3.45 \mathrm{~nm}$ in the refined structure.

In order to probe the influence of the alkyl chain length on the resulting framework, we synthesized a short-chain analogue of the a4T building block bearing two methyl groups instead of the butyl chains. Due to the significantly lower solubility of this $\mathrm{a}_{4} \mathrm{~T}_{\mathrm{Me}}$ building block, the COF synthesis required a 2.5 times more dilute reaction mixture. The resulting framework is isostructural to the original a4T COF, with a slightly smaller unit cell (SI, Figure S15).

Under identical synthetic conditions as those chosen for the Py-a4T COF and under all other solvent combinations and concentrations tested, the reaction between Py and 2 equiv of s4T yielded only orange-red, fully amorphous networks. This observation confirms our above conclusions drawn from the structure modeling and highlights the importance of the building block design.

The asymmetric modification strategy also allows us to extend one of the thiophene units into a more-electrondeficient fused heterocycle, thus forming a donor-acceptor (D-A) building block with modified optical and electronic properties while retaining the overall geometry of the $4 \mathrm{~T}$ backbone.

Incorporation of a single $4 H$-thieno[3,4-c]pyrrole-4,6 $(5 H)$ dione (TPD) or thieno[3,4-b] thiophene (TT) via sequential Stille coupling reactions yielded the $4 \mathrm{~T}_{\mathrm{TPD}}$ and $4 \mathrm{~T}_{\mathrm{TT}}$ building blocks, respectively (Figure 3a; see the SI for experimental details). For the best possible crystallinity, the $4 \mathrm{~T}_{\mathrm{TT}}$ building block was synthesized as an isomer-pure material with the orientation of the TT subunit as shown in Figure 3a. In both cases, the asymmetric incorporation enables the $4 \mathrm{~T}$ derivatives to stack in a close-packed alternating sequence despite the sterically demanding acceptor moieties (Figure 3b,c).

The acid-catalyzed reaction of Py with 2 equiv of the modified 4T building blocks under solvothermal conditions yielded the $\mathrm{Py}-4 \mathrm{~T}_{\mathrm{TPD}}$ and $\mathrm{Py}-4 \mathrm{~T}_{\mathrm{TT}} \mathrm{COFs}$, respectively. The formation of crystalline frameworks was confirmed by PXRD analysis (Figure 3d,e). Pawley refinements of the Py-4T $\mathrm{T}_{\mathrm{TPD}}$ and Py- $4 \mathrm{~T}_{\mathrm{TT}}$ COFs using the force-field-optimized structure models displayed as insets in Figure 3d,e reproduced the experimental patterns very well. Following the same reasoning as for the simulation and refinement of the Py-a4T COF (see above), the asymmetric $4 \mathrm{~T}_{\mathrm{TPD}}$ and $4 \mathrm{~T}_{\mathrm{TT}}$ building blocks were approximated as a superposition of both possible orientations with $50 \%$ occupancy each. The refined unit cell parameters are $a=5.16 \pm$ $0.05 \mathrm{~nm}, b=5.30 \pm 0.05 \mathrm{~nm}, c=0.384 \pm 0.02 \mathrm{~nm}$, and $\beta=72^{\circ}$ $\pm 5^{\circ}$ for the Py-4T $\mathrm{T}_{\mathrm{TPD}} \mathrm{COF}$ and $a=4.76 \pm 0.05 \mathrm{~nm}, b=5.10$ $\pm 0.05 \mathrm{~nm}, c=0.384 \pm 0.02 \mathrm{~nm}$, and $\beta=72^{\circ} \pm 5^{\circ}$ for the Py$4 \mathrm{~T}_{\mathrm{TT}}$ COF. The longer pore diagonals $a$ and $b$ in these COFs compared to the Py-a4T COF can be attributed to a slight variation in the optimal stacking offset perpendicular to the $4 \mathrm{~T}$ axis and the resulting differences in the tilt of the $4 \mathrm{~T}$ columns versus the pyrene core. The length of the crystallographic $c$ axis, on the other hand, is dominated by the stacking of the pyrene units and, thus, hardly affected by the tilt of the $4 \mathrm{~T}$ bridge. 
a

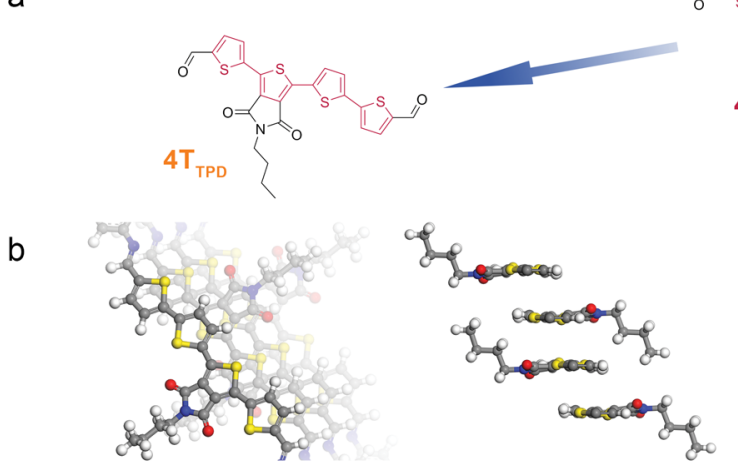

d

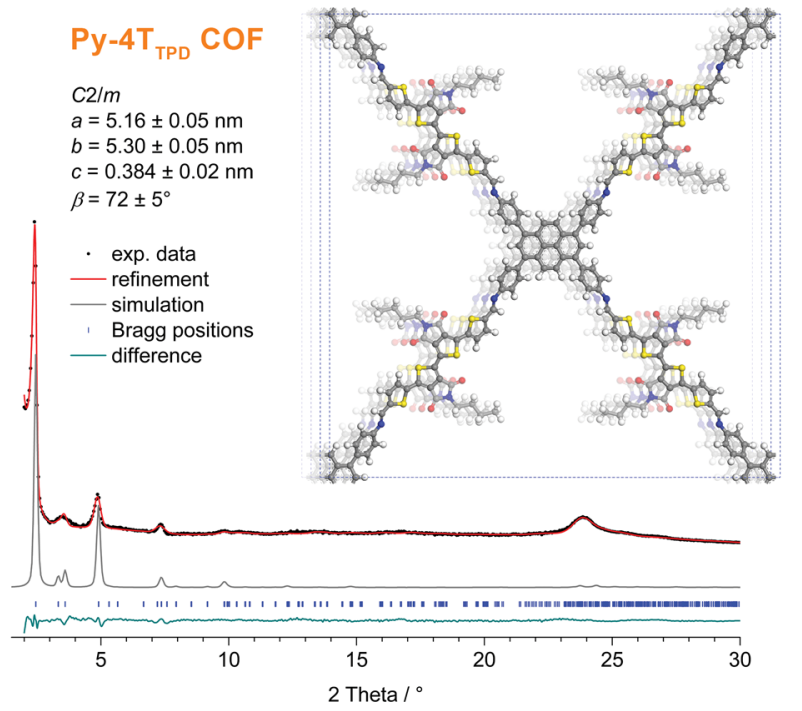

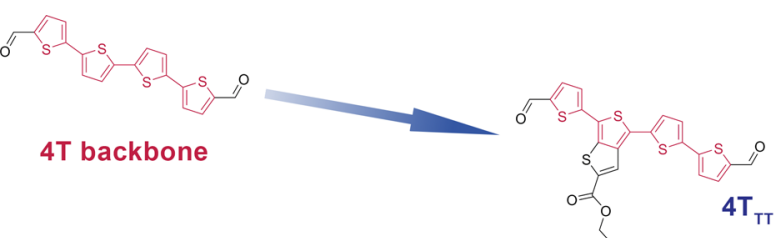

C

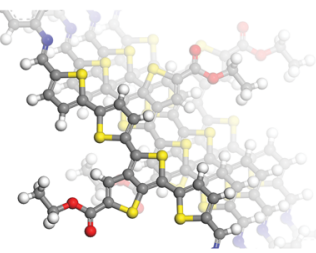

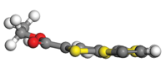

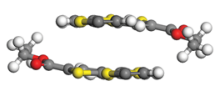

coosengege

e

$\mathrm{Py}-4 \mathrm{~T}_{\mathrm{TT}}$ COF

$\mathrm{C} 2 / \mathrm{m}$

$a=4.76 \pm 0.05 \mathrm{~nm}$ $b=5.10 \pm 0.05 \mathrm{~nm}$ $c=0.384 \pm 0.02 \mathrm{~nm}$ $\beta=72 \pm 5^{\circ}$

$\mid$\begin{tabular}{c} 
- exp. data \\
- refinement \\
- simulation \\
\hline - Bragg position
\end{tabular}

Bragg positions

- difference

a

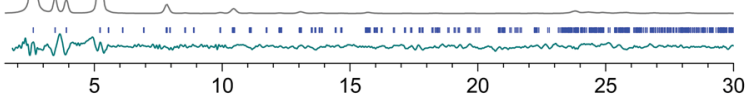

2 Theta ${ }^{\circ}$

Figure 3. (a) The asymmetric modification approach can also be used to construct quaterthiophene-based donor-acceptor building blocks, in which one thiophene is replaced by an electron-deficient 3,4-extended thiophene. (b, c) Despite the increased steric demands of these subunits, a closepacked cofacial arrangement of the asymmetric building blocks is possible via alternating orientation of the electron-deficient moieties. (d, e) Experimental PXRD data (black dots) of the Py- $4 \mathrm{~T}_{\mathrm{TPD}}$ and Py- $4 \mathrm{~T}_{\mathrm{TT}}$ COFs, respectively, and corresponding Pawley-refined patterns (red lines) and difference plots (green lines; $R_{\mathrm{wp}}=5.2 \%, R_{\mathrm{p}}=14.3 \%$ and $R_{\mathrm{wp}}=9.3 \%, R_{\mathrm{p}}=16.1 \%$, respectively). Bragg positions are indicated with blue ticks. The acceptor moieties (TPD and TT) were treated in the refinements as a superposition of both possible orientations with $50 \%$ occupancy each (both shown in the representations of the unit cells). The simulated PXRD patterns (gray lines) based on the $\mathrm{C} 2 / \mathrm{m}$ symmetric unit cells (insets) agree very well with the experimental and refined patterns of the respective frameworks. Differences in the peak intensities, in particular the altered intensity ratios between lower- and higher-angle reflections, might stem from unreacted precursor materials or small fragments adsorbed in the COF pores (the attenuation of peak intensities due to this is strongest at small $2 \theta$ angles).
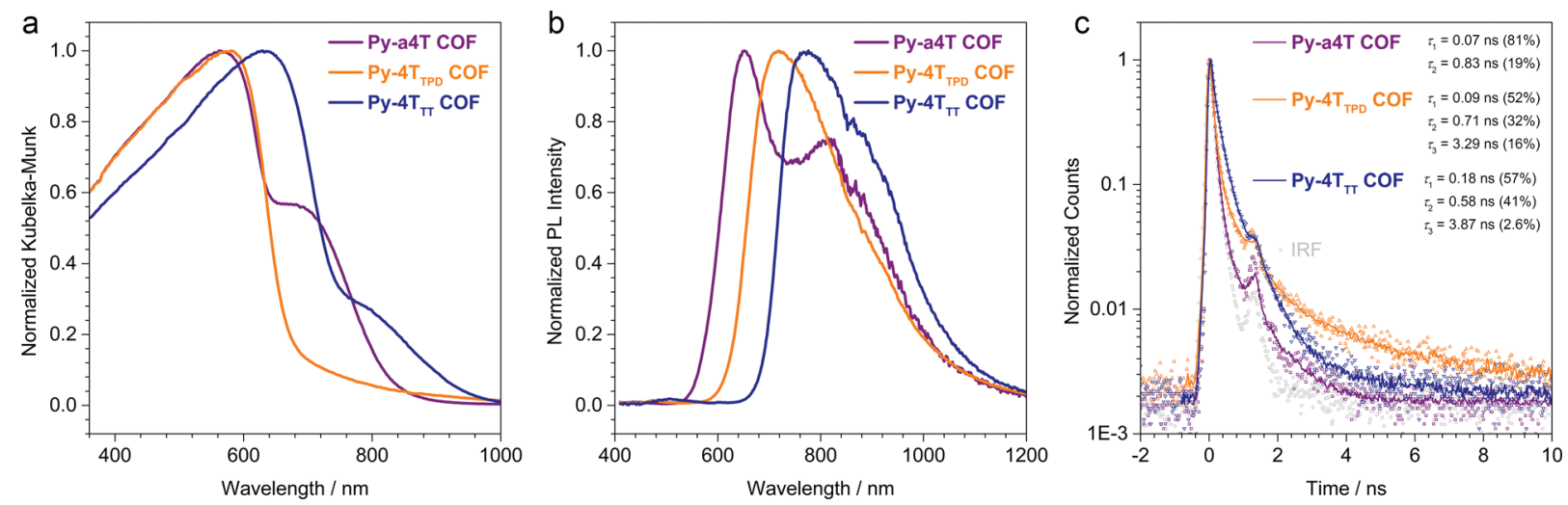

Figure 4. (a) UV-vis-NIR absorption of the three thiophene-bridged COFs, calculated from the diffuse reflectance spectra of the COF powders dispersed in $\mathrm{BaSO}_{4}$. (b) PL spectra measured with pulsed $378 \mathrm{~nm}$ excitation and (c) the corresponding TCSPC traces recorded at the respective emission maximum of each COF. The lines represent exponential deconvolution fits taking into account the instrument response function and scattering (see the SI for details; the rise at $1.35 \mathrm{~ns}$ originates from the diode laser). The COFs exhibit fast biexponential (Py-a4T) or triexponential $\left(\mathrm{Py}-4 \mathrm{~T}_{\mathrm{TPD}}\right.$ and $\left.\mathrm{Py}-4 \mathrm{~T}_{\mathrm{TT}}\right)$ decays with sub-nanosecond components. The fractional intensities, i.e., the fractions of collected photons corresponding to the respective lifetimes, are stated in brackets. 
The new 4T-based COFs are intensely colored powders that are capable of absorbing most of the visible spectrum (Figure 4a). The optical band gaps estimated from Tauc plots are 1.57, 1.17 (indirect), and $1.40 \mathrm{eV}$ for the Py-a4T, Py-4T $\mathrm{TPD}_{\mathrm{T}}$, and Py$4 \mathrm{~T}_{\mathrm{TT}}$ COFs, respectively (SI, Figure S8). Electronic integration of the $4 \mathrm{~T}$ subunit is apparent from the strong red-shift of the absorption in comparison to previously reported pyrene COFs with small acene bridges. Furthermore, the increased absorption capabilities of the D-A building blocks (SI, Figures S6 and S7) translate to red-shifted absorption maxima of the corresponding COFs (Py-a4T COF, $563 \mathrm{~nm}$; Py-4T $\mathrm{T}_{\mathrm{TPD}} \mathrm{COF}$, $\left.580 \mathrm{~nm} ; \mathrm{Py}-4 \mathrm{~T}_{\mathrm{TT}} \mathrm{COF}, 638 \mathrm{~nm}\right)$. All three COFs feature an additional absorption band below the energy of the main $\pi-\pi^{*}$ transition that is relatively weak for the $\mathrm{Py}-4 \mathrm{~T}_{\mathrm{TPD}}$ and strongest for the Py-a4T COF. This might indicate the formation of a charge transfer state between the electron-rich pyrene and the slightly electron-deficient 4T subunits (see below).

The photoluminescence (PL) spectra follow the same trends as the absorption spectra with emission maxima of the COFs at 654, 718, and $773 \mathrm{~nm}$, respectively (Figure 4b and SI, Figures S9 and S10).

The hypothesis of a photoinduced charge transfer between the two imine-linked building blocks is supported by the timecorrelated single photon counting (TCSPC) traces of the COFs (Figure 4c). Dilute solutions of the 4T building blocks exhibit monoexponential decay curves with lifetimes of about $0.5 \mathrm{~ns}\left(\mathrm{a} 4 \mathrm{~T}, 4 \mathrm{~T}_{\mathrm{TT}}\right)$ or $1 \mathrm{~ns}\left(4 \mathrm{~T}_{\mathrm{TPD}}\right)$ (SI, Figures $\mathrm{S} 12$ and $\left.\mathrm{S} 13\right)$. In the solid state, the PL lifetimes of the pure building blocks are increased to $\tau_{1} \sim 0.5 \mathrm{~ns}$ and $\tau_{2} \sim 1 \mathrm{~ns}$ (biexponential decays). The Py building block shows even longer lifetimes of above $2 \mathrm{~ns}$ in solution and a main component of $>1 \mathrm{~ns}$ in the solid state. If integrated into a COF, however, the PL lifetimes are drastically reduced. The fastest component, which represents more than $50 \%$ of the emitted photons, drops to below 200 ps $\left(\mathrm{Py}_{-} 4 \mathrm{~T}_{\mathrm{TT}}\right)$ or even below 100 ps (Py-a4T, Py$\left.4 \mathrm{~T}_{\mathrm{TPD}}\right)$. This indicates that the imine-linked pairing with the tetraphenylpyrene has opened up a new pathway for fast and efficient conversion of the COF $\pi^{*}$ excited state, potentially via electron transfer to the quaterthiophenes and hole transfer to the pyrene. This is further supported by comparison with the PL lifetimes reported for electron-rich acene-bridged pyrene COFs that do not enable strong intramolecular charge transfer. ${ }^{15}$ In these materials, the lifetimes are much longer, ranging from $0.35 \mathrm{~ns}$ to well above $1 \mathrm{~ns}$.

The existence of an efficient charge transfer pathway that competes effectively with the radiative recombination of the COF $\pi^{*}$ excited state is additionally supported by the quenching of the $4 \mathrm{~T} \mathrm{PL}$ upon incorporation into the COF (SI, Figure S11). This effect is most significant for the Py-a4T $\mathrm{COF}$, which is consistently the material that exhibits the strongest charge transfer absorption band in the diffuse reflectance spectrum. To the best of our knowledge, this is the first observation of the formation of a charge transfer state between the subunits of an imine-linked COF.

\section{CONCLUSION}

In this work, we have developed the first quaterthiophenebased $2 \mathrm{D}$ covalent organic frameworks comprising ordered $\pi$ stacked columns of $4 \mathrm{~T}$ and pyrene moieties. Following an asymmetric functionalization strategy of the otherwise $C_{2}$ symmetric $4 \mathrm{~T}$ backbone allowed us to incorporate alkyl chains for optimized solubility while fully retaining the ability of the building blocks to form close-packed face-on stacked thiophene columns. We demonstrate that this strategy also provides a facile route for modifying the electronic properties of the $4 \mathrm{~T}$ backbone via incorporation of electron-deficient subunits, thus forming donor-acceptor type molecules. The absorption and emission spectra confirm that the $4 \mathrm{~T}$-based building blocks are electronically integrated into the framework. Spectral features below the energy of the $\pi-\pi^{*}$ transition and the analysis of the corresponding emission decay time traces reveal the fast and efficient formation of a charge transfer state between the iminelinked pyrene and quaterthiophene subunits. We believe that our new asymmetric building block design provides a general strategy for the construction of well-ordered COFs from various extended building blocks. This will greatly expand the range of applicable molecules for realizing frameworks with tailor-made optoelectronic properties.

\section{ASSOCIATED CONTENT}

\section{S Supporting Information}

The Supporting Information is available free of charge on the ACS Publications website at DOI: 10.1021/jacs.7b01631.

Experimental methods, synthetic procedures, and additional structural and spectroscopic data (PDF)

\section{AUTHOR INFORMATION}

\section{Corresponding Authors}

*fa355@cam.ac.uk

*bein@lmu.de

ORCID $\odot$

Florian Auras: 0000-0003-1709-4384

\section{Notes}

The authors declare no competing financial interest.

\section{ACKNOWLEDGMENTS}

The authors are grateful for funding from the German Science Foundation (DFG; Research Cluster NIM) and the Free State of Bavaria (Research Network SolTech). The research leading to these results has received funding from the European Research Council under the European Union's Seventh Framework Programme (FP7/2007-2013)/ERC Grant Agreement No. 321339. The authors thank Dr. Markus Döblinger for the transmission electron microscopy.

\section{REFERENCES}

(1) Côte, A. P.; Benin, A. I.; Ockwig, N. W.; O’Keeffe, M.; Matzger, A. J.; Yaghi, O. M. Science 2005, 310, 1166.

(2) Furukawa, H.; Yaghi, O. M. J. Am. Chem. Soc. 2009, 131, 8875.

(3) Doonan, C. J.; Tranchemontagne, D. J.; Glover, T. G.; Hunt, J. R; Yaghi, O. M. Nat. Chem. 2010, 2, 235.

(4) Stegbauer, L.; Schwinghammer, K.; Lotsch, B. V. Chem. Sci. 2014, $5,2789$.

(5) Lin, S.; Diercks, C. S.; Zhang, Y.-B.; Kornienko, N.; Nichols, E. M.; Zhao, Y.; Paris, A. R.; Kim, D.; Yang, P.; Yaghi, O. M.; Chang, C. J. Science 2015, 349, 1208.

(6) Chandra, S.; Kundu, T.; Kandambeth, S.; BabaRao, R.; Marathe, Y.; Kunjir, S. M.; Banerjee, R. J. Am. Chem. Soc. 2014, 136, 6570.

(7) Shinde, D. B.; Aiyappa, H. B.; Bhadra, M.; Biswal, B. P.; Wadge, P.; Kandambeth, S.; Garai, B.; Kundu, T.; Kurungot, S.; Banerjee, R. J. Mater. Chem. A 2016, 4, 2682.

(8) Dogru, M.; Handloser, M.; Auras, F.; Kunz, T.; Medina, D.; Hartschuh, A.; Knochel, P.; Bein, T. Angew. Chem., Int. Ed. 2013, 52, 2920. 
(9) Guo, J.; Xu, Y.; Jin, S.; Chen, L.; Kaji, T.; Honsho, Y.; Addicoat, M. A.; Kim, J.; Saeki, A.; Ihee, H.; Seki, S.; Irle, S.; Hiramoto, M.; Gao, J.; Jiang, D. Nat. Commun. 2013, 4, 2736.

(10) Calik, M.; Sick, T.; Dogru, M.; Döblinger, M.; Datz, S.; Budde, H.; Hartschuh, A.; Auras, F.; Bein, T. J. Am. Chem. Soc. 2016, 138, 1234.

(11) Ascherl, L.; Sick, T.; Margraf, J. T.; Lapidus, S. H.; Calik, M.; Hettstedt, C.; Karaghiosoff, K.; Döblinger, M.; Clark, T.; Chapman, K. W.; Auras, F.; Bein, T. Nat. Chem. 2016, 8, 310.

(12) Bunck, D. N.; Dichtel, W. R. Angew. Chem., Int. Ed. 2012, 51, 1885.

(13) Shinde, D. B.; Kandambeth, S.; Pachfule, P.; Kumar, R. R.; Banerjee, R. Chem. Commun. 2015, 51, 310.

(14) Chen, L.; Furukawa, K.; Gao, J.; Nagai, A.; Nakamura, T.; Dong, Y.; Jiang, D. J. Am. Chem. Soc. 2014, 136, 9806.

(15) Auras, F.; Ascherl, L.; Hakimioun, A. H.; Margraf, J. T.; Hanusch, F. C.; Reuter, S.; Bessinger, D.; Döblinger, M.; Hettstedt, C.; Karaghiosoff, K.; Herbert, S.; Knochel, P.; Clark, T.; Bein, T. J. Am. Chem. Soc. 2016, 138, 16703.

(16) Lin, G.; Ding, H.; Yuan, D.; Wang, B.; Wang, C. J. Am. Chem. Soc. 2016, 138, 3302-3305.

(17) Wan, S.; Gándara, F.; Asano, A.; Furukawa, H.; Saeki, A.; Dey, S. K.; Liao, L.; Ambrogio, M. W.; Botros, Y. Y.; Duan, X.; Seki, S.; Stoddart, J. F.; Yaghi, O. M. Chem. Mater. 2011, 23, 4094.

(18) Jin, S.; Ding, X.; Feng, X.; Supur, M.; Furukawa, K.; Takahashi, S.; Addicoat, M.; El-Khouly, M. E.; Nakamura, T.; Irle, S.; Fukuzumi, S.; Nagai, A.; Jiang, D. Angew. Chem., Int. Ed. 2013, 52, 2017.

(19) Calik, M.; Auras, F.; Salonen, L. M.; Bader, K.; Grill, I.; Handloser, M.; Medina, D. D.; Dogru, M.; Löbermann, F.; Trauner, D.; Hartschuh, A.; Bein, T. J. Am. Chem. Soc. 2014, 136, 17802.

(20) Cai, S.-L.; Zhang, Y.-B.; Pun, A. B.; He, B.; Yang, J.; Toma, F. M.; Sharp, I. D.; Yaghi, O. M.; Fan, J.; Zheng, S.-R.; Zhang, W.-G.; Liu, Y. Chem. Sci. 2014, 5, 4693.

(21) Kim, J. Y.; Kim, S. H.; Lee, H.-H.; Lee, K.; Ma, W.; Gong, X.; Heeger, A. J. Adv. Mater. 2006, 18, 572.

(22) Irwin, M. D.; Buchholz, D. B.; Hains, A. W.; Chang, R. P. H.; Marks, T. J. Proc. Natl. Acad. Sci. U. S. A. 2008, 105, 2783.

(23) Cnops, K.; Rand, B. P.; Cheyns, D.; Verreet, B.; Empl, M. A.; Heremans, P. Nat. Commun. 2014, 5, 3406.

(24) Kan, B.; Li, M.; Zhang, Q.; Liu, F.; Wan, X.; Wang, Y.; Ni, W.; Long, G.; Yang, X.; Feng, H.; Zuo, Y.; Zhang, M.; Huang, F.; Cao, Y.; Russell, T. P.; Chen, Y. J. Am. Chem. Soc. 2015, 137, 3886.

(25) Li, Z.; He, G.; Wan, X.; Liu, Y.; Zhou, J.; Long, G.; Zuo, Y.; Zhang, M.; Chen, Y. Adv. Energy Mater. 2012, 2, 74.

(26) Zhang, Q.; Kan, B.; Liu, F.; Long, G.; Wan, X.; Chen, X.; Zuo, Y.; Ni, W.; Zhang, H.; Li, M.; Hu, Z.; Huang, F.; Cao, Y.; Liang, Z.; Zhang, M.; Russell, T. P.; Chen, Y. Nat. Photonics 2015, 9, 35.

(27) Bertrand, G. H. V.; Michaelis, V. K.; Ong, T.-C.; Griffin, R. G.; Dinca, M. Proc. Natl. Acad. Sci. U. S. A. 2013, 110, 4923.

(28) Medina, D. D.; Werner, V.; Auras, F.; Tautz, R.; Dogru, M.; Schuster, J.; Linke, S.; Döblinger, M.; Feldmann, J.; Knochel, P.; Bein, T. ACS Nano 2014, 8, 4042.

(29) Medina, D. D.; Rotter, J. M.; Hu, Y.; Dogru, M.; Werner, V.; Auras, F.; Markiewicz, J. T.; Knochel, P.; Bein, T. J. Am. Chem. Soc. 2015, 137, 1016.

(30) Feldblyum, J. I.; McCreery, C. H.; Andrews, S. C.; Kurosawa, T.; Santos, E. J. G.; Duong, V.; Fang, L.; Ayzner, A. L.; Bao, Z. Chem. Commun. 2015, 51, 13894.

(31) Lohse, M. S.; Rotter, J. M.; Margraf, J. T.; Werner, V.; Becker, M.; Herbert, S.; Knochel, P.; Clark, T.; Bein, T.; Medina, D. D. CrystEngComm 2016, 18, 4295.

(32) Rabbani, M. G.; Sekizkardes, A. K.; Kahveci, Z.; Reich, T. E.; Ding, R; El-Kaderi, H. M. Chem. - Eur. J. 2013, 19, 3324.

(33) Chen, X.; Huang, N.; Gao, J.; Xu, H.; Xu, F.; Jiang, D. Chem. Commun. 2014, 50, 6161. 